\section{Actividad antibacteriana de Caesalpinia spinosa (tara) sobre Porphyromonas gingivalis}

\author{
Antibacterial activity of Caesalpinia spinosa (tara) on Porphyromonas \\ gingivalis
}

\begin{abstract}
Resumen
Objetivo: Determinar la actividad antibacteriana in vitro de cinco concentraciones $(6,25 ; 12,5 ; 25 ; 50$ y 75 $\mathrm{mg} / \mathrm{mL}$ ) del extracto alcohólico de la Caesalpinia spinosa "tara" (EACS) sobre Porphyromonas gingivalis. Materiales y método: El estudio es de tipo experimental, prospectivo, comparativo e in vitro, y se llevó a cabo en el Laboratorio de Microbiología de la Facultad de Odontología de la UNMSM. Se usó el método de difusión en placa para enfrentarlas a las soluciones del extracto alcohólico de Caesalpinia spinosa y compararlas con el control positivo Clorhexidina $0,12 \%$ y control negativo Alcohol $96^{\circ}$. Resultados: Se determinó que la concentración del extracto alcohólico de Caesalpinia spinosa tiene efecto antibacteriano sobre Porphyromonas gingivalis, aunque el aumento de la concentración no guarda una relación proporcional con el aumento de diámetro del halo de inhibición. El análisis estadístico mediante la prueba de Kruskall- Wallis determinó que no existen diferencias significativas entre las diferentes concentraciones del EACS y mediante la prueba de Mann - Whitney se determinó que tampoco existen diferencias significativas entre el EACS y el control positivo Clorhexidina $0,12 \%$ y el control negativo Alcohol $96^{\circ}$. Conclusiones: Se ha evidenciado el efecto antibacteriano sobre Porphyromonas gingivalis. Palabras clave: Caesalpinia spinosa, Actividad antibacteriana, Test de difusión en placa, Porphyromonas gingivalis.
\end{abstract}

Artículo Original

\section{Álex Montenegro Chipana', Donald Ramos Perfecto ${ }^{2}$}

1: Escuela Académico Profesional de la Facultad de Odontología de la UNMSM.

2: Departamento de Ciencias Básicas de la Facultad de Odontología de la UNMSM.

Correspondencia:

CD. Alex Montenegro Chipana

Calle Faustino Sarmiento 251 Puente Piedra Lima-Perú

Correo electrónico: odosm@hotmail.com Coautor: dramos_37@hotmail.com

\begin{abstract}
Objective: Determine the antibacterial activity in vitro of five concentrations $(6.25,12.5,25,50$ and 75 $\mathrm{mg} / \mathrm{mL}$ ) of the alcoholic extract of Caesalpinia spinosa "tara" (EACS) on Porphyromonas gingivalis. Materials and method: The study is experimental, prospective, comparative in vitro and kind, was carried out in the Laboratory of Microbiology, Faculty of Dentistry, San Marcos. Diffusion method was used in plate solutions to confront the alcoholic extract of Caesalpinia spinosa and compared with the positive control chlorhexidine $0.12 \%$ and Alcohol $96{ }^{\circ}$ negative control. Results: It was found that the concen-tration of the alcoholic extract of Caesalpinia spinosa has antibacterial effect on Porphyromonas gingivalis, although the increased concentration bears no proportion to the increased diameter of the inhibition zone. Statistical analysis using the Kruskal-Wallis test found no significant differences between different concentrations of EACS and by the Mann - Whitney determined that there are no significant differences between the EACS and the positive control chlorhexidine $0.12 \%$ and control Alcohol $96^{\circ}$ negative. Conclusions: We have demonstrated the antibacterial effect on Porphyromonas gingivalis. Keywords: Caesalpinia spinosa, Antibacterial activity, Disk diffusion test, Porphyromonas gingivalis.
\end{abstract}

Fecha de recepción: 10/11/2015

Fecha de aceptación: 10/05/2016

\section{Introducción}

Desde tiempos remotos, la población del mundo ha recurrido a las plantas con la finalidad de curar o aliviar alguna dolencia, transmitiéndose sus beneficios de generación en generación sin conocer la acción de sus principios activos. En el Perú se han realizado estudios experimentales de diferentes plantas medicinales, entre ellas la Caesalpinia spinosa (C. spinosa) "tara", llegando a la conclusión de algunas propiedades de sus componentes, entre ellas ser antibacteriana, antihemorrágica, analgésica, antiinflamatoria, etc.

El uso de plantas medicinales se mantiene en vigencia a través de los años, teniendo, en los últimos años, un rol importante como fuente de medicamentos en zonas rurales. ${ }^{1}$

De acuerdo a la Organización Mundial de la Salud (OMS), una planta medicinal es definida como cualquier espe- cie vegetal que contenga sustancias que puedan ser empleadas con propósitos terapéuticos y cuyos principios activos puedan servir de precursores en la síntesis de nuevos fármacos. ${ }^{2}$ Dichas plantas presentan una propiedad curativa en virtud a los metabolitos que elaboran, y para que estas nos proporcionen los efectos deseados se debe ingerir sus principios activos, es decir, aquellos componentes que contengan un determinado poder curativo. ${ }^{3}$

(c) Los autores. Este artículo es publicado por la Revista Odontología Sanmarquina de la Facultad de Odontología, Universidad Nacional Mayor de San Marcos. Este es un artículo de acceso abierto, distribuido bajo los términos de la licencia Creative Commons Atribucion - No Comercia_Compartir Igual 4.0 Internacional. (http://creativecommons.org/licenses/by-nc-sa/4.0/) que permite el uso no comercial, distribución y reproducción en cualquier medio, siempre que la obra original sea debidamente citada. Para uso comercial, por favor póngase en contactto con revista.odontologia@gmail.com. 
El uso empírico de la tara para el tratamiento de afecciones de las vías respiratorias nos permite inferir que esta planta presenta propiedades antibacterianas sobre los microorganismos que la causen, lo que constituirá un recurso alternativo. ${ }^{4}$

El Perú es el país que tiene mayor área de bosques de tara, con el $80 \%$ de la producción mundial. Se encuentra en los valles interandinos secos entre 1000 y $3100 \mathrm{msnm}$, siendo los departamentos de mayor producción Cajamarca (41\%), Ayacucho (16\%). ${ }^{5}$

Porphyromonas gingivalis (P. gingivalis) es un importante miembro de la microbiota periodontal, ${ }^{6,7}$ involucrado tanto en la progresión de la periodontitis, como en los procesos de destrucción de hueso y tejido blando. ${ }^{8-10}$ También, puede producir severas infecciones extraorales, incluyendo mediastinales, de planos faciales, cerebro y abscesos pulmonares, ${ }^{9}$ enfermedades cardiacas, ${ }^{10}$ parto prematuro y bajo peso al nacer. $^{11,12}$

En investigaciones realizadas "in vitro" con extracto de C. spinosa "tara" se ha demostrado que tiene actividad antibacteriana contra Staphylococcus aureus, Escherichia coli, Pseudomonas aeruginosa, Klebsiella sp. y Shiguella flexnerii y otras realizadas "in vivo" ha demostrado su actividad antiinflamatoria. ${ }^{13}$

$\mathrm{Liu}^{14}$ realizó estudios de actividad antibacteriana in vitro de los extractos de las vainas y semillas de C. spinosa utilizando cepas Gram positivas (Staphylococcus aureus y Bacillus subtilis) y Gram negativas (Escherichia coli, Klebselia sp. y Shigella flexneri) mediante técnica de difusión con disco. Los extractos fueron preparados usando como solvente alcohol-acetona (1:1). Se observó actividad inhibitoria sobre cepas Gram positivas para el extracto de la vaina de tara, mas no para el de la semilla.

En estudios realizadas por De La Cruz ${ }^{15}$ se determinó el efecto del extracto hidroalcohólico de C. spinosa (Molina) Kuntze "tara" sobre la viabilidad de Streptococcus $\beta$ hemolítico. Encontrándose que la actividad antibacteriana del extracto de C. spinosa frente a Streptococcus $\beta$ hemolítico aumenta a medida que se eleva (25\% a $100 \%)$ la concentración del extracto.

La $P$. gingivalis es una bacteria de relevancia en las enfermedades periodontales, la cual ha sido enfrentada con otros productos naturales obteniendo resultados prometedores ${ }^{16}$. Esto motivó a realizar el presente trabajo para conocer el efecto antibacteriano del extracto de C. spinosa "tara" sobre P. gingivalis, a fin de proporcionar una alternativa en el tratamiento de infecciones periodontales, que complemente el tratamiento odontológico, sabiendo que estos son económicos y están al alcance de los que tienen menos recursos.

\section{Materiales y método}

Para la realización del estudio, se usó la cepa ATCC 33277 de P. gingivalis previamente identificadas por los laboratorios MICROBIOLOGIC.

La preparación de las cinco concentraciones se realizó en la Facultad de Farmacia y Bioquímica de la UNMSM, CENPROFARMA. Se usó vainas de C. spinosa (tara) limpiadas y desinfectadas con cloro al $1 \%$ y colocadas en un cuarto de secado por 48 horas, luego se procedió a separar las semillas de las vainas colocándolas en un mortero para su pulverizado, se pesó 50 gr. de polvo de las vainas y se colocó en un frasco de vidrio de color ámbar y se le agregó $200 \mathrm{ml}$ de alcohol $70^{\circ}$, dejándose macerar por 1 semana, agitándolo todos los días. $\mathrm{Al} 4^{\text {to }}$ día se agregó 100 $\mathrm{ml}$ más de alcohol y se siguió agitando hasta completar la semana. El producto fue filtrado 3 veces, con papel filtro Whatmann No 41, No 2 y No 1 , obteniéndose un extracto purificado. Luego se colocó en un frasco de vidrio de color ámbar y fue secado por liofilización. A partir de esta masa de extracto de C. spinosa "tara" se prepararon concentraciones de 6,$25 ; 12,5 ; 25 ; 50$ y $75 \mathrm{mg} / \mathrm{ml}$, las cuales fueron guardadas en frascos de color ámbar estériles y conservadas en refrigeración.

Una vez adquirida las cepas puras, estas fueron reactivadas en una placa de agar Schadler suplementado con sangre de cordero al $5 \%$ para microorganismos anaerobios. Este paso se realizó en un tiempo no mayor de 5 minutos. Luego se colocó en una jarra de anaerobiosis para ser incubado por 7 a 10 días. (Figura 1). Una vez reactivada la cepa, se diluyó en un tubo de ensayo con $2 \mathrm{ml}$ de agua bidestilada hasta obtener una turbidez de Mac Farland de 0,5. Se prepararon las soluciones experimentales y los controles.
El test de sensibilidad se llevó por medio de la prueba de difusión en agar para lo cual se utilizó el medio de agar Schadler suplementado con sangre de cordero al $5 \%$, que se sembró por diseminación con un hisopo estéril (el cual se realizó de manera uniforme sobre la placa). Luego se colocaron los discos de papel, ya embebidos con las diluciones del extracto, en las placas de agar .La siembra de las soluciones se realizó por quintuplicado. Se realizó el transporte inmediato de las placas hacia la jarra de anaerobiosis (con sobres de anaerobiosis) esperando la formación de halo de inhibición (Figura 2) alrededor de los discos en el agar, incubando en condiciones a $37^{\circ} \mathrm{C}$ por siete días.

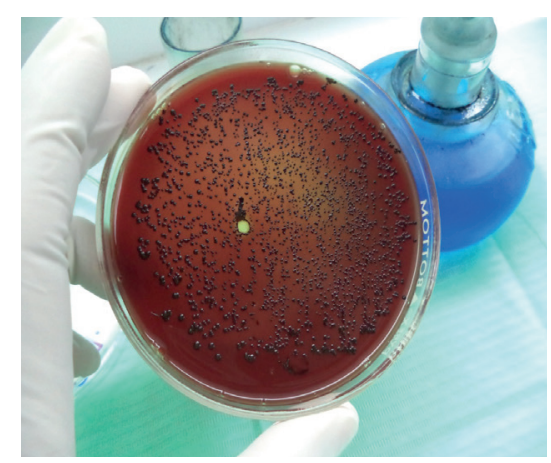

Fig. 1. Colonias de Porphyromonas gingivalis.

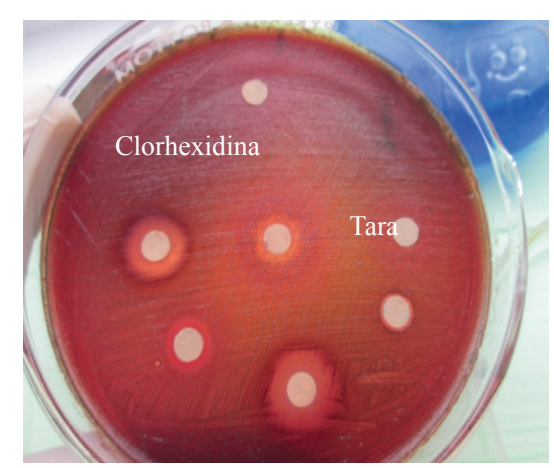

Fig. 2. Test de difusión en placa, obsérvese los halos de inhibición.

Para el análisis del efecto antibacteriano de las soluciones de $C$. spinosa frente a $P$. gingivalis se consideró en función al diámetro de los halos de inhibición del crecimiento del microorganismo: nula (-) si fue inferior o igual a $8 \mathrm{~mm}$; sensibilidad límite (sensible + ) de 9 a 14 mm; media ( sensible ++ ) de 15 a 19 mm y sumamente sensible (S.S. +++$)$ si fue igual o superior a $20 \mathrm{~mm}$. (Según aromatograma de Duraffourd). ${ }^{17}$ 


\section{Resultados}

Los resultados mostraron que el extracto alcohólico de C.spinosa tiene actividad antibacteriana in vitro sobre P. gingivalis, siendo la media de los diámetros de los halos de inhibición 13,$50 ; 10,00 ; 12,50 ; 12,50$ y 7,00 correspondiendo el mayor promedio para la concentración de $12,5 \mathrm{mg} / \mathrm{ml}$ y la menor concentración para la de $75 \mathrm{mg} /$ $\mathrm{ml}$ (Figura 3).

En la Figura 3 se puede apreciar que el efecto antibacteriano de C. Spinosa sobre $P$. gingivalis no es directamente proporcional a su concentración. El análisis estadístico mediante la prueba de Kruskall- Wallis indicó que no existen diferencias significativas $(\mathrm{p}, 0,05)$ entre las diferentes concentraciones de C. spinosa y al realizar el análisis de los resultados del grupo experimental (extractos de $C$. spinosa) y el grupo control, mediante la prueba U Mann Whitney, se determinó que no existe una diferencia estadísticamente significativa $(\mathrm{p}, 0,05)$.
En la Tabla $\mathrm{N}^{\circ} 1$ se muestra la actividad antibacteriana del extracto de $C$. spinosa sobre Porphyromonas gingivalis, donde se observa que los halos de inhibición del crecimiento bacteriano se encuentran en mayor cantidad en el rango de sensibilidad límite (8-14mm) que corresponden a la formación de 11 halos (tres halos corresponden a la concentración de $12,5 \%$ y cuatro halos al control positivo) y que la concentración de $12,5 \%$ nunca estuvo en el rango de sensibilidad nula (‘ $8 \mathrm{~mm})$ (según el aromatograma de Duraffourd).

\section{CONCENTRACIÓN}

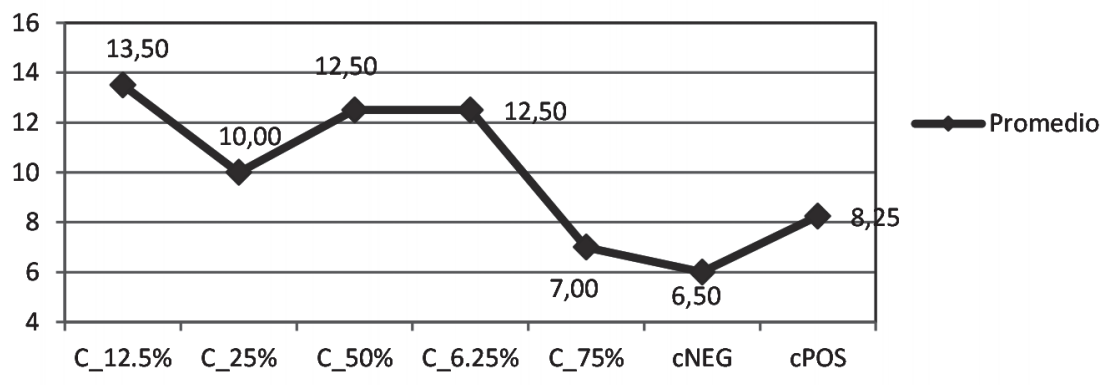

Fig. 3. Efecto antibacteriano de las concentraciones de Caesalpinia spinosa sobre P. gingivalis.

Tabla 1. Concentraciones en $\mathrm{mg} / \mathrm{ml}$ de la C. spinosa sobre P. gingivalis

\begin{tabular}{lccccccc}
\hline Rango de sensibilidad & \multicolumn{4}{c}{$\begin{array}{c}\text { Concentraciones del extracto } \\
\text { alcoholico de c. spinosa }\end{array}$} & $\begin{array}{c}\text { Clorhexidina } \\
0,12 \%\end{array}$ & $\begin{array}{c}\text { Alcohol } \\
96 \%\end{array}$ \\
\cline { 2 - 6 } & 75 & 50 & 25 & 12,5 & 6,25 & & $\mathrm{n} \%$ \\
Nula: $<8 \mathrm{~mm}$ & $\mathrm{n} \%$ & $\mathrm{n} \%$ & $\mathrm{n} \%$ & $\mathrm{n} \%$ & $\mathrm{n} \%$ & $\mathrm{n} \%$ \\
Límite: $8-14 \mathrm{~mm}$ & 250 & 125 & 125 & 000 & 125 & 000 & 4100 \\
Media:15-19mm & 250 & 250 & 250 & 375 & 250 & 4100 & 000 \\
Sumamente sensible $: \geq 20 \mathrm{~mm}$ & 000 & 000 & 125 & 000 & 000 & 000 & 000 \\
Total & 000 & 150 & 000 & 125 & 125 & 000 & 000 \\
\hline & 4100 & 4100 & 4100 & 4100 & 4100 & 4100 & 4100 \\
\hline
\end{tabular}

\section{Discusión}

La C. spinosa "tara" posee dentro de su composición química taninos, flavonoides, péptidos, compuestos fenólicos, lo que se evidencia en el trabajo realizado por López, ${ }^{18}$ el cual hizo un estudio de las propiedades antimicrobianas de la tara de diversas regiones del Perú, encontrando que la acción antimicrobiana de la tara varía por su procedencia pero que los taninos, flavonoides y péptidos tenían la propiedad antimicrobiana.

En trabajos realizados por Lannacone ${ }^{19}$ y Añanca ${ }^{11}$ utilizaron para sus investigaciones extractos acuosos de C. spinosa. Lannacone ${ }^{19}$ lo usó a una concentración del $20 \%$ sobre Sitophilus zeamais Moyts- chulsky y Stegobium paniceum no encontrando efecto significativo y Añanca ${ }^{11}$ lo usó a concentraciones de 17,$5 ; 16,25$; $15 ; 13,75 ; 12,5 ; 11,25 ; 10 ; 8,75$ y 6,25 $\mu \mathrm{g} / \mathrm{ml}$, en cepas de Staphylococcus aureus y Streptococcus pyogenes determinando actividad antibacteriana in vitro. Para este trabajo se usó extracto alcohólico de C. spinosa a concentraciones de $75 \% ; 50$ $\%, 25 \% ; 12,5 \% ; 6,25 \%$ tal como lo usara Huarino, ${ }^{20}$ quien en su estudio demostró la actividad antibacteriana de C. spinosa a las mismas concentraciones de este trabajo, demostrando actividad antibacteriana sobre flora salival mixta. Escobar $^{21}$ utilizó extracto alcohólico de C.spinosa sobre Corynebacteium diphtheriae usando inóculos estandarizados con el Nefelómetro de Mac Farland
No 0,5 encontrando diámetros de inhibición que varían de 34,11 a 43,55 y que a mayor concentración aumenta el diámetro de halo de inhibición. El presente estudio se llevó a cabo sobre cepas de $P$. gingivalis, principal patógeno en la periodontitis. Se utilizó la cepa ATCC 33277, tal como lo usara Ramos, ${ }^{16}$ el cual investigó la actividad antibacteriana, in vitro, del extracto de Erythroxylum coca, sobre la cepa ATCC de $P$. gingivalis, mediante el test de difusión en agar. Los resultados indicaron que el extracto de Erythroxylum coca tiene sensibilidad límite para la máxima concentración del extracto (100\%), el cual genera halos de inhibición entre 8 y 14 mm. Para el enfrentamiento se usó el test de difusión con disco, encontran- 
do la presencia de halos de inhibición en las cinco concentraciones, los cuales superan al control negativo (alcohol). Esta técnica fue usada por Liu ${ }^{15}$ encontrando actividad inhibitoria sobre cepas Gram positivas. Escobar, ${ }^{20}$ utilizando esta misma técnica, demostró que a mayor concentración se obtiene mayor halo de inhibición sobre Corynebacterium diphtheriae. De la misma manera, Huarino ${ }^{20}$ demostró mediante esta misma técnica que el efecto antibacteriano de C. spinosa sobre flora salival mixta es directamente proporcional a su concentración.

Este trabajo de investigación permite un alcance preliminar al determinar in vitro que el extracto de $C$. spinosa tiene actividad antibacteriana sobre $P$. gingivalis, evaluado mediante el test de difusión en agar con discos. El efecto antibacteriano del extracto alcanzó un rango de actividad entre 8 y $25 \mathrm{~mm}$ sobre $P$. gingivalis. La mayor actividad se da con la concentración de $12,5 \%$ y la menor actividad con la de $75 \%$; sin embargo, estas diferencias no fueron significativas. Se demostró que cada una de las concentraciones del extracto tiene actividad antibacteriana, aunque no existe diferencia significativa entre ellas. Los valores de los halos de inhibición no guardan una relación proporcional con las concentraciones del extracto. Se observó que el control positivo (Clorhexidina $0,12 \%$ ) presenta actividad antibacteriana sobre $P$. gingivalis. Los resultados obtenidos demuestran su actividad antibacteriana con formación de halos de inhibición que varían entre 8 y $9 \mathrm{~mm}$ y una media de $8,25 \mathrm{~mm}$.

\section{Conclusiones}

1. La concentración del extracto alcohólico de C. spinosa $(6,25 \mathrm{mg} / \mathrm{ml} ; 12,5$ $\mathrm{mg} / \mathrm{ml} ; 25 \mathrm{mg} / \mathrm{ml} ; 50 \mathrm{mg} / \mathrm{ml} \mathrm{y} 75 \mathrm{mg} /$ $\mathrm{ml} 6,25 \mathrm{mg} / \mathrm{ml}$ a $75 \mathrm{mg} / \mathrm{ml}$ ) tiene efecto antibacteriano sobre Porphyromonas gingivalis, aunque el aumento de la concentración no guarda una relación proporcional con el aumento de diámetro del halo de inhibición. La concentración de $12,5 \mathrm{mg} / \mathrm{ml}$ mostró mejor sensibilidad antibacteriana que el control positivo (Clorhexidina al $0,12 \%$ ) colutorio de elección en el apoyo del tratamiento de procesos periodontales.

\section{Agradecimientos}

Al Q.F. Fritz Choquesillo Peña, Director de CENPROFARMA de la Facultad de Farmacia y Bioquímica de la UNMSM, por la elaboración y la realización de los análisis cualitativos del extracto de C. spinosa.

\section{Referencias bibliográficas}

1. Garrido VH. Efecto Antimicrobiano de la Caesalpinia spinosa (TARA) y tetraciclina frente Actinobacillus actinomycetemcomitans. [Tesis para obtener el Título de Cirujano Dentista]. Lima: Fac de Odontol,Universidad San Martín de Porres; 2003

2. Oliveira M, Velásquez D, Bermúdez A. La investigación etnobotánica sobre plantas medicinales: una revisión de sus objetivos y enfoques actuales. 2005; 30(8): 453-459.

3. Waizel-Bucay J, Martinez I. Plantas empleadas en odontalgias I. Medigraphic (serial de internet). 2006 Jul. 10 (citado: 2007 Set.Oct) 64(5): 173-186. Disponible en: http://www.medigraphic.com/ pdfs/adm/od-2007/od075.pdf

4. Agapito T, Sung I. Plantas Medicinales. $7 \mathrm{ma}$ ed. Lima-Perú: Isabel. 2000:53.Pag

5. Cabello Liu I. Monografía para el cultivo de la tara Caesalpinia Spinosa (Molina) Kuntze. Perúbiodiverso (serial de internet). Lima, Perú. 2009:5. Disponible en: http://perubiodiverso.pe/assets/ Monograf\%C3\%ADa-del-cultivode-la-tara1.pdf

6. Ishikawa I, Baheni P. Nonsurgical periodontal therapy - where do we stand now? Periodontol 2000. 2004; 36: 9-13.

7. Ramos PD, Moromi NH, Martinez CE. Porphyromonas gingivalis: patógeno relevante en la periodontitis crónica. Odontol Sanmarquina. 2011; 14(1): 34-38.

8. Holt S, Kesavalu L, Walker S. \& Genco C. Virulence factors of Porphyromonas gingivalis. Periodontol 2000.1999;20:168-238.
9. Genco RJ, Zambon JJ, and Christersson LA. The origin of periodontal infection. Adv Dent Res.1998; 2(2): 245-259.

10. Beck J, Offenbacher S. Periodontits: A risk factor coronary heart disease?. Ann of Periodontol .1998; 3: 127-141.

11. Añanca E. Efecto antibacteriano in vitro del extracto acuoso de vainas de Caesalpinia spinosa (tara) en cepas de Staphylococcus aureus y Streptococcus pyogenes. [Tesis para optar el Título de Químico Farmacéutico]. Tacna: Fac Ciencias Médicas Escuela Académico Profesional de Farmacia y Bioquímica, Universidad Nacional Jorge Basadre Grohmann;2009

12. Gamonal JA, López NJ, Aranda W. Periodontal conditions and treatment needs, by CPITN, in the 35-44 and 65-74 years old population in Santiago, Chile. Int Dent J. 1998; 48: 96-103.

13. Infantes AY. Tratamiento de la gingivitis marginal crónica con pasta dental de Caesalpinia spinosa (Molina) KUNTZE “TARA” en niños de 8 a 10 años. [Tesis para optar el Título de Cirujano Dentista]. Lima: Facultad de Odontología Universidad San Martín de Porres; 2004.

14. Liu H, Lengual L, León G, La Torre C, Huapaya J, Chauca J. Evaluación de la Actividad Antibacteriana in vitro de los Extractos de Caesalpinia spinosa "tara" y Eucalyptus sp. "eucalipto”. Rev Horiz Med. 2002; 2(1-2):40-44.

15. De La Cruz M. Efecto del extracto hidroalcohólico de Caesalpinia spinosa (MOLINA) Kuntze "Tara" sobre la viabilidad de Streptococcus $\alpha$-hemolítico. [Tesis Maestral]. Trujillo: Facultad de Farmacia y Bioquímica: UNT; 2009.

16. Ramos CA. Actividad antibacteriana del extracto de Erythroxylum coca sobre Porphyromonas gingivalis, estudio in vitro. [Tesis Maestral]. Trujillo: Facultad de Farmacia y Bioquímica: UNT; 2009. 
17. Alzamora L, Morales L, Armas L, Fernández G. Medicina tradicional en el Perú actividad antimicrobiana in vitro de los aceites esenciales extraídos de algunas plantas aromáticas. An Fac med. 2001; 62(2):156-161

18. López FC. Acción antimicrobiana Caesalpinia tintórea (Molina) Kuntze o Tara de diferentes regiones del Perú. Rev CLEIBA.1998; $1(1): 27-31$.
19. Iannacone J, Ayala H, Román A. Efectos Toxicológicos de Cuatro Plantas sobre el Gorgojo del Maíz Sitophlus zeamais Motschlsky y sobre el Gorgojo de las Galletas Stegobium paniceum en Perú. Rev Gayana. 2005; 69(2):234-240.

20. Huarino M. Efecto Antibacteriano de Caesalpinia Spinosa (Tara) Sobre Flora Saliva Mixta. Tesis para optar el Título de Cirujano Den- tista. Fac de Odontol: UNMSM. Lima. 2011.

21. 21. Escobar Bl. Efecto in vitro de diferentes concentraciones de extracto alcohólico de Caesalpinia spinosa (Molina) Kuntze, sobre la viabilidad de Corynebacterium diphtheriae. Rev Med Vallejiana. 2008; 5(1):28-37. 